\title{
Antimicrobial resistance in Klebsiella pneumoniae as an independent risk factor for bacteraemia-related mortality
}

Hong Kong Med J 2021;27:385

https://doi.org/10.12809/hkmj219773

To the Editor-Although many research endeavours focus on the microbiology, epidemiology, and molecular characterisation of extended-spectrum beta-lactamase-producing and carbapenemresistant Gram-negative bacteria, few studies aim to assess the impact of these resistance traits on patient outcomes. Therefore, Man et $\mathrm{al}^{1}$ should be applauded for linking antimicrobial resistance in Klebsiella pneumoniae strains with the risk of inappropriate empirical treatment and infection-related mortality. ${ }^{1}$ The role of empirical antibiotics in septic patients was also highlighted as a key consideration. However, although extended-spectrum betalactamase-producing or carbapenem-resistant $K$ pneumoniae isolates were associated with a greater risk of inappropriate empirical treatment, and subsequently with significantly higher 90-day and hospital mortalities, the manuscript would benefit from delineating these two groups of resistant bacteria, as well as from including Pitt bacteraemia scores. Moreover, a paramount study by Patel et $\mathrm{al}^{2}$ showed that even appropriate empirical treatment is often not associated with improved survival among patients with carbapenem-resistant $K$ pneumoniae infections. Also, heteroresistance is an under-recognised phenomenon that may render K pneumoniae strains resistant to antibiotics (despite in vitro susceptibility) and, in turn, confound any steadfast conclusions. ${ }^{3}$ This is why linking patient-level microbiology data with clinical records and patient outcomes in different settings will be a priority in years to come, as evidenced by trailblazing Global Research on AntiMicrobial resistance (GRAM) Project led by the Institute for Health Metrics and Evaluation (University of Washington) and the Big Data Institute (University of Oxford). ${ }^{4}$ The highest burden of sepsis-related deaths was already demonstrated in locations least equipped to identify or treat $\operatorname{sepsis}^{5}$; thus, going forward, studies akin to Man et $\mathrm{al}^{1}$ analysing individual-level data will be indispensable.

\begin{abstract}
Author contributions
The author had full access to the data, contributed to the letter, approved the final version for publication, and takes responsibility for its accuracy and integrity.
\end{abstract}

\section{Conflicts of interest}

The author has no conflicts of interest to disclose.

\section{Acknowledgement}

The author is involved in the Global Research on AntiMicrobial resistance (GRAM) Project, Institute for Health Metrics and Evaluation, University of Washington, Seattle, United States.

\section{Funding/support}

This letter received no specific grant from any funding agency in the public, commercial, or not-for-profit sectors.

1,2 T Meštrović * $\mathrm{MD}, \mathrm{PhD}$

${ }^{1}$ Clinical Microbiology and Parasitology Unit, Dr Zora Profozić Polyclinic, Zagreb, Croatia

2 University Centre Varaždin, University North, Varaždin, Croatia

*Corresponding author: tmestrovic@unin.hr

\section{References}

1. Man MY, Shum HP, Li KC, Yan WW. Impact of appropriate empirical antibiotics on clinical outcomes in Klebsiella pneumoniae bacteraemia. Hong Kong Med J 2021;27:24757.

2. Patel G, Huprikar S, Factor SH, Jenkins SG, Calfee DP. Outcomes of carbapenem-resistant Klebsiella pneumoniae infection and the impact of antimicrobial and adjunctive therapies. Infect Control Hosp Epidemiol 2008;29:1099-106.

3. Band VI, Weiss DS. Heteroresistance: a cause of unexplained antibiotic treatment failure? PLoS Pathog 2019;15:e1007726.

4. Schnall J, Rajkhowa A, Ikuta K, Rao P, Moore CE. Surveillance and monitoring of antimicrobial resistance: limitations and lessons from the GRAM project. BMC Med 2019;17:176.

5. Rudd KE, Johnson SC, Agesa KM, et al. Global, regional, and national sepsis incidence and mortality, 1990-2017: analysis for the Global Burden of Disease Study. Lancet 2020;395:200-11. 